\title{
Multi-episodic perceived quality for one session of consecutive usage episodes with a speech telephony service
}

\author{
Dennis Guse $^{1}\left[\right.$ Benjamin Weiss $^{1}{ }^{1} \cdot$ Frank Haase $^{1} \cdot$ Anna Wunderlich $^{1}$. \\ Sebastian Möller ${ }^{1}$
}

Received: 13 December 2016/Published online: 3 August 2017

(c) Springer International Publishing AG 2017

\begin{abstract}
The impact of varying performance and its effect on the perceived quality is an important aspect of quality of experience. Especially for service providers, it is important to understand how the perceived quality of a user, who is interacting with their services repeatedly, evolves. Repeateduse of a service is actually common for telecommunication services, such as speech telephony. For telecommunication services, it is very likely that a user encounters varying performance, for example due to varying network load conditions. For repeated-use taking place in a usage period covering several days, initial work on the formation process of the so-called multi-episodic perceived quality (i.e., distinct, meaningful interactions, denoted as usage episodes) has been conducted. In this paper, we present our work investigating the multi-episodic perceived quality for usage episodes taking place in one session (i.e., in a time frame of up to $45 \mathrm{~min}$ ) with a speech telephony service. We investigate two aspects and their impact on the multi-episodic perceived quality: (a) increasing the number of low performance (LP) usage episodes and (b) varying the temporal position of LP usage episodes towards the assessment of multi-episodic perceived quality. Additionally, the impact of
\end{abstract}

Dennis Guse

dennis.guse@alumni.tu-berlin.de

Benjamin Weiss

benjamin.weiss@tu-berlin.de

Frank Haase

fra.haase@gmail.com

Anna Wunderlich

a.wunderlich@campus.tu-berlin.de

Sebastian Möller

sebastian.moeller@tu-berlin.de

1 Technische Universität Berlin, Berlin, Germany the user's behavioral freedom (i.e., two-party conversation vs. 3rd-party listening) on the two investigated aspects is examined. With regard to (a), the results show that an increasing number of LP usage episodes lead to a reduced multi-episodic perceived quality until saturation is reached. No impact of the user's behavioral freedom was found. For (b), an impact of the varied behavioral freedom was observed. Varying the temporal position had an impact on the multi-episodic perceived quality for two-party conversation. For 3rd-party-listening, this could only be observed in one out of two cases.

Keywords Quality of experience - Episodic usage $\cdot$ Recall of repeated usage $\cdot$ Retrospective judgments

\section{Introduction}

For speech telecommunication services, the perceived quality is an important aspect as it is expected to influence the current behavior and the future behavior of a user. This includes how a user engages a service to solve his task(s), the attitude towards the service, and also whether the service will be used again. This user-centric notion is embedded in the concept of quality of experience (QoE), which "is the degree of delight or annoyance of a person whose experiencing involves an application, service, or system. It results from the person's evaluation of the fulfillment of his or her expectations and needs with respect to the utility and / or enjoyment in the light of the person's context, personality and current state" [32]. The formation process of perceived quality is considered a continuous process integrating the continuous stream of experiences [21]. This process can be investigated in subjective experiments by presenting a set of stimuli to participants 
and letting them assess their perceived quality. Here, judgments can be taken momentarily (i.e., while experiencing) or in retrospection (i.e., after an experience) [44]. In difference to a momentary judgment, a retrospective judgment is based upon memorized information that can later be recalled for this judgment. It is thus affected by the underlying processes of memorization and recall.

For the investigation of perceived quality in case of speech telephony, typically short stimuli (usually in the range of $8-12 \mathrm{~s}$ ) as well as individual telephone calls are assessed. The former focuses on the precise evaluation of degradations including perception thresholds (e.g., comparison and enhancement of speech coding algorithms) while the latter focuses on the assessment in ecologically valid situations. With regard to the perceived quality, repeated-use has been rarely investigated, which is, however, the common case (i.e., a user is interacting with the same service repeatedly over time to fulfill his needs) [6]. For repeated-use, a user forms an impression of the multiepisodic perceived quality about all his individual interactions with a service. Although the characteristics of the formation process of multi-episodic perceived quality are not (yet) fully understood, it is expected that judgments of multi-episodic perceived quality are affected by cognitive biases that have been observed for retrospective judgments of individual experiences.

The goal of the here presented research was to investigate the formation process in detail and thus establish a solid basis for future work in terms of definitions as well as data sets. In this paper, we present the results of two experiments investigating the formation process of multi-episodic perceived quality. For these experiments, the case of multiple consecutive usage episodes in one session (i.e., up to $45 \mathrm{~min}$ overall duration) was selected. This complements initial work on multi-episodic perceived quality in which episodic usage over several days was investigated [10, 11, 29]. In the two experiments, three aspects were examined: (i) the impact of an increasing number of severely degraded usage episodes, (ii) the impact of varying the temporal position of severely degraded usage episodes, and (iii) the impact of the two different usage situations (i.e., interactive vs. passive use). This research was conducted using a speech telephony service, as speech telephony is well-investigated with regard to perceived quality.

This paper is structured as follows: ${ }^{1}$ First, we present our definition of usage episode, which is inspired by the concept of episodic memory as well as task-driven usage. Subsequently, an overview on known biases affecting

\footnotetext{
1 The here presented work is also included in the dissertation of Guse [9]. With this paper, we provide an overview on the current state of research on multi-episodic perceived quality and hopefully stimulate future research in this direction.
}

retrospective judgments is given, and the state of the art on multi-episodic perceived quality is presented. Following, the two conducted experiments are presented in "Subjective experiments" and the results are analyzed in "Data analysis". Based upon the results, we present an approach towards predicting multi-episodic judgments in "Prediction of the final multi-episodic judgment". This paper closes with a discussion of the results and gives an outlook for future work on multi-episodic perceived quality for telecommunication services.

\section{Perceived quality for multi-episodic usage}

\section{Usage episode and retrospective judgments}

We define usage episode as a distinct and self-contained interaction by a user with a service or system to achieve his or her goal(s). Goal achievement as requirement for a usage episode follows the concept of utility and expected utility by Kahneman [22]. In fact, an evaluation of a usage episode can only be done in retrospection (i.e., after a usage episode is finished). Therefore, an evaluation must be based upon information that has been memorized while experiencing. Following the notation of episodic memory [41], a usage episode groups together experiences by their spatio-temporal relationship (i.e., time, location, goal, emotions, and situation). In fact, only a reduced representation of the information can be memorized and thus might be available later for recall. A person recalling an episode is able to re-experience it by re-creating a representation of the past reality based upon recallable information about this specific episode [41]. For a retrospective evaluation of an episodic experience, only these pieces of information can be used that are recallable at the time of the evaluation. This might not encompass all memorized pieces of information and might even contain pieces of information not belonging to the episode to be recalled [37]. With regard to the evaluation in terms retrospective judgments of individual episode(s), it was shown that not all parts of an episode are equally important $[1,22]$. For retrospective evaluation of physical pain, Kahneman et al. [23] observed a recency effect (i.e., later experiences in an episode have a higher impact on the retrospective judgment than earlier experiences). Also, it has been observed that exceptional parts of an episode have a higher impact on a retrospective judgment. This is denoted as peak effect [22]. Moreover, the so-called duration neglect has been observed. Here, the duration of exceptional parts of an episode [22] as well as the duration of an episode $[1,22]$ are neglected to a certain degree (i.e., longer parts/episodes are judged similar to shorter parts/episodes with similar characteristics). The reason why not all parts of an episode are equally taken 
into account for a retrospective judgment might be found in the difficulty to recall. Information that is easier to recall seems to be considered more relevant for retrospective judgments regardless of the reason for the easier recall (e.g., temporal closeness or importance) [28].

\section{Perceived quality under varying performance}

In the domain of perceived quality, the impact of noticeably varying performance on retrospective judgments has been investigated mainly for video transmission and speech telephony. ${ }^{2}$ Here, similar effects to retrospective judgments of general experiences were investigated and observed (i.e., mainly recency effect and peak effect). Fundamental work was conducted by Hands and Avons [14] based on the beliefadjustment model [15]. This model explains the occurrence of the recency effect and the duration neglect depending upon the integration process of new information into one's belief. For $30 \mathrm{~s}$ video sequences, Hands and Avons could observe a recency effect (i.e., presenting similar degradations earlier results in a lower impact on retrospective judgments) as well as a duration neglect (i.e., presenting either 5 or $10 \mathrm{~s}$ of reduced performance yielded similar retrospective judgments). For video sequences of up to $180 \mathrm{~s}$ duration, Hamberg and de Ridder [13] observed a recency effect and a peak effect while varying the duration of impairments from 2 to $10 \mathrm{~s}$. With regard to shorter stimuli, effects on retrospective judgments tend to be unobservable (e.g., Ninassi et al. [30] for $8 \mathrm{~s}$ video sequences). For (speech) telephony, mainly recency was investigated and observed (e.g., [2, 7, 8, 13, 27, 34, 43]). Also a peak effect was observed (e.g., [2, 27, 43]). In addition, work has also been conducted with regard to the perception of waiting times. This has been conducted for web browsing as well as multimedia streaming. An overview on this topic is given by Egger et al. [4]. In this domain it could be observed that an initial delay (i.e., at the beginning of an interaction) is perceived different than later interruptions. In retrospective judgments, an initial delay is judged better than interruptions. This is likely to be explained by the interruption of the flow during an ongoing interaction. Shaikh et al. [39] found for consecutive web pages that increasing the delay immediately reduces following retrospective judgments. If the delay is decreased afterwards again, following retrospective judgments remain affected. Similar observations were made by Sackl et al. [36] and Hoßfeld et al. [16].

For the investigation of perceived quality, identical or very similar stimuli are assessed by multiple observers to derive the judgment of the average observer (cf. [32]). The judgment of the average observer is usually reported in terms of a Mean Opinion Score (MOS). The assessment of

\footnotetext{
$\overline{2}$ In this paper only effects on retrospective judgments are discussed. For an overview on momentary judgments, we recommend [44].
}

the perceived quality for telecommunication services is challenging as varying user behavior might affect the formation process of perceived quality. Nevertheless, varying user behavior needs to be considered to derive ecological valid findings. For speech telephony, this means to expose a participant to a conversation or a similar, realistic situation. In fact, conversations consist of alternating listening parts and speaking parts for individual participants. The exact repetition with regard to content and speaker changes is rather unlikely, which is an issue for deriving a MOS. This is especially problematic for the assessment of varying transmission performance as the impact on perceived quality might be affected by the conversational flow. To overcome this limitation, the method of simulated conversations has been developed and standardized by ETSI [5]. Here, a conversation is completely defined. Each participant must follow this definition while switching between the predefined listening parts and speaking parts. This allows presenting very similar stimuli, most importantly including timing of degradations, to multiple participants and thus deriving a MOS. This method was applied for speech-only telephony [27, 43] and also video telephony [2]. In fact, this method limits the behavioral freedom of participants while trying to recreate a realistic situation. Although this approach might introduce undesired effects, it allows to investigate the impact of varying performance on perceived quality using a limited number of participants and thus provides an economical benefit. For investigations on web browsing, similar approaches were used defining the exact order of web pages to be visited (e.g., [40]).

\section{Excursus: investigation of multi-episodic usage in user experience}

A different facet of multi-episodic usage is investigated in the field of user experience (UX). QoE and UX conceptually overlap as both focus on experiences with technology (cf. [42]). UX, stemming from usability, focuses on the interaction with technology. Investigating multi-episodic usage is an important aspect of UX, as user behavior as well as emotions towards technology tend to change over time. This might be due to acquiring knowledge about technical capabilities and limitations. For UX, the concept of multi-episodic usage is described by Roto et al. [35, p. 8].

While multi-episodic usage is not a key aspect for UX, it is an important aspect especially regarding the adaptation of new products and new services. Initial work on multiepisodic usage with regard to UX was empirically conducted by Karapanos et al. [24] and Kujala et al. [25]. Here, participants used a smartphone and reported how their expectations, their usage behavior, and their satisfaction 
changed. It was observed that the usage and emotions towards the product under investigation changed over time. In the beginning, interactions were more playful and exploratory, but latter became more task-oriented and productive. Kujala et al. [25] presented and applied the $U X$ curve method to retrospectively assess remembered changes towards the product under investigation. Here, a participant draws a line reflecting how his satisfaction changed over time. He annotates the vertices with the reasons for the changes and includes recallable adaptations of his usage behavior. Although the assessment is conducted in retrospect and thus likely affected by memory-related effects, this method allows to measure changes in user behavior and emotions over time efficiently.

\section{Prior work on multi-episodic perceived quality}

The formation process of perceived quality over several usage episodes with one service has so far only received limited attention. First work on the so-called multi-episodic perceived quality was conducted by Duncanson [3] for a speech telephony service. He assessed (a) the perceived quality of a just finished call with the actual average performance of the service and (b) the assumed perceived quality of an imagined call with the subjective average performance of the service. It could be observed that judgments for (a) yielded a higher score than (b). Duncanson concludes "that ratings of single, recent telephone calls yield results different from ratings of subjectively averaged, past telephone calls of the same type". However, the reason(s) for the observed difference could not be derived and were left for future work that has not yet been conducted.

\section{Introducing the terminology and the defined-use method}

In difference to Duncanson [3], in which the service could be used freely (i.e., real users interacted with the telephony service when desired to solve their individual tasks), Möller et al. [29] applied the defined-use method. ${ }^{3}$ In the defined-use method a set of usage episodes is defined that need to be conducted with the service under investigation. Here, it is specified when, for what purpose, and how the service under investigation has to be used. For each usage episode, a task is supplied in order to accomplish realistic, meaningful usage. If very similar tasks are supplied, this allows to achieve a comparable usage behavior over a subset or all usage episodes. In the defined-use method, a multi-episodic condition defines the set of usage episodes. This also includes the performance for each individual usage episode. Presenting one multi-episodic condition to multiple participants allows to derive the MOS of the resulting multi-episodic perceived

\footnotetext{
${ }^{3}$ The term defined-use method was introduced in Guse [12, p. 22].
}

quality. The impact of the varying performance on the multiepisodic MOS can be evaluated by purposefully varying only the performance of usage episodes between otherwise identical multi-episodic conditions. For the evaluation of multiple multi-episodic conditions, a between-subject design must be considered to avoid an impact of the presentation order of multi-episodic conditions on the derived judgments. While the defined-use method inherently limits ecological validity as participants are forced to solve the defined usage episodes, this method enables reproducibility. Table 1 gives an overview on prior work on multi-episodic perceived quality applying the defined-use method.

\section{Experiments covering multiple days}

Möller et al. [29] applied the defined-use method to investigate multi-episodic perceived quality with a video telephony service (i.e., Skype). In this experiment, pairs of participants conducted one multi-episodic condition (12 days; two usage episodes per day; usage in the participant's home environment). A usage period of 12 days was chosen allowing participants to familiarize with the service. For each usage episode, the task was to solve one Short Conversation Scenario (SCS) (i.e., ITU-T Recommendation P.805 [18]). These two-party telephony scenarios suggest a conversational structure while a typical telephone-related task needs to be solved. In this experiment, the role of caller and callee was alternated. After each usage episode, the perceived quality of this usage episode was assessed. The multi-episodic perceived quality was assessed after the $2 \mathrm{nd}, 7 \mathrm{th}$, and 12 th day. These are in the following denoted as episodic judgments and multiepisodic judgments, respectively. Within each usage episode, Möller et al. [29] opted for applying non-varying performance to avoid macroscopic fluctuations [31, p. 72]. This avoids potential still unknown effects due to performance variations during one usage episode on episodic judgments and multi-episodic judgments. For each usage episode, the performance was defined by limiting the unidirectional transmission bandwidth for caller and callee. This affected the audio transmission channel as well as the video transmission channel. Although the different applied transmission bandwidths were reported, the precise parameters resulting in the performance levels were not included. Möller et al. [29] applied five multi-episodic conditions. Each multi-episodic condition contained only High Performance (HP) and Low Performance (LP). ${ }^{4,5} \mathrm{HP}$

\footnotetext{
${ }^{4}$ Please note that the parameters of HP and LP are throughout this paper specific to each experiment although the same notation is used.

5 Möller et al. [29] also used a third performance level denoted as medium performance. However, medium performance was only presented in one multi-episodic condition in which HP was not presented and thus took the role of HP.
} 
Table 1 Overview on prior experiments on multi-episodic perceived quality applying the defined use-method

\begin{tabular}{|c|c|c|}
\hline & Multi-episodic conditions & Service(s) (usage pattern in brackets) \\
\hline Möller et al. [29] & 5 à 12 days & Video telephony (twice per day) \\
\hline Guse and Möller [10] & 2 à 14 days & $\begin{array}{l}\text { Speech telephony (once per day) } \\
\text { Video-on-demand (once per day) }\end{array}$ \\
\hline Guse et al. [11] & 2 à 14 days & $\begin{array}{l}\text { Audio-on-demand (once per day) } \\
\text { Video-on-demand (once per day) }\end{array}$ \\
\hline Guse et al. [12] & 2 à 6 usage episodes (one session) & Audio-on-demand (sequential use) \\
\hline
\end{tabular}

and LP were selected to be clearly distinguishable. In the five investigated multi-episodic conditions, at least the first four usage episodes were presented in HP. This allowed participants to familiarize with the highest performance of the service and form an internal reference that the later presented usage episodes could be compared to. Participants were recruited in pairs knowing each other before the experiment and required to have prior experiences with Skype. The episodic judgments showed that the defineduse method could be applied successfully in a field experiment (i.e., the selected performance levels resulted in consistent episodic judgments). With regard to multi-episodic judgments, the results were limited as slight, mainly non-significant, differences within each multi-episodic condition as well as between multi-episodic conditions were indicated only. The reason might be found in the small difference between HP and LP or in the low number of LP usage episodes per multi-episodic condition.

Subsequently, the defined-use method was applied by Guse and Möller [10] for a video-on-demand service and a speech telephony service. They followed closely the design of Möller et al. [29]. Here, two multi-episodic conditions were applied with a usage period of 14 days presenting usage episodes in HP and LP. However, the speech telephony service failed to provide the defined performance levels. Thus, participants were not exposed to the same multi-episodic condition, and a multi-episodic MOS could not be derived. The video-on-demand service functioned as desired, but the results were similar to Möller et al. [29] (i.e., limited impact of LP usage episodes on multi-episodic judgments).

Based upon these findings, Guse et al. [11] focused on more severe, unrealistic degradations and increased the number of LP usage episodes per multi-episodic condition. In this experiment, an audio-on-demand service and a video-on-demand service needed to be used once per day over a usage period of 14 days. The applied performance levels resulted, as desired, in a reduction of subsequent multi-episodic judgments. After an initial drop due to the LP usage episodes, the multi-episodic judgments, which were collected every 3 days, remained on a similarly low level. Even three consecutive HP usage episodes resulted only in a slight, non-significant increase of the directly following multi-episodic judgment. However, it was found that the very first LP usage episode had a lower impact on multi-episodic judgments. This indicates that the very first encounter with a LP usage episode is judged differently.

\section{Experiments for one session}

Based on the limited findings of experiments with usage periods covering several days, Guse et al. [12] presented initial work on multi-episodic perceived quality in one session (i.e., several consecutive usage episodes). In the presented experiment, the impact of varying duration of LP usage episodes on episodic judgments and multi-episodic judgments was investigated. In this experiment, an audioon-demand service presenting a speech-only audio book was used for six usage episodes. Two multi-episodic conditions were applied presenting the 5th usage episode in LP. Usage episodes had a duration of approximately $3 \mathrm{~min}$. Between the two multi-episodic conditions only the duration of the 5 th usage episode was varied (3 vs. 6 min). The results showed that varying the duration of the 5th usage episode yielded neither a significant difference for the episodic judgment nor for the multi-episodic judgment. This indicates that a duration neglect occurred (i.e., the duration of a usage episode is not reflected in its retrospective judgment). This is in line with findings on retrospective judgments of general experiences. Furthermore, this suggests that multi-episodic judgments might be based on episodic judgments alone.

\section{Prediction of multi-episodic judgments}

First approaches towards the prediction of multi-episodic judgments based on prior episodic judgments were undertaken by Möller et al. [29]. Here, the unweighted average of all prior episodic judgments was evaluated. While this model works well for earlier multi-episodic judgments, the prediction accuracy decreases for later multi-episodic judgments. Guse et al. [11] could improve the prediction accuracy by accounting for a recency effect using the results of their reported experiment and the results of Möller et al. [29]. They evaluated a windowed unweighted 
average and a linear weighted average. It was found that the linear weighted average achieves a higher robustness against non-optimal parameter selection. Accounting for a peak effect as described by Weiss et al. [43] did not improve the prediction accuracy. It must be noted that Guse et al. [11] and Möller et al. [29] evaluated the prediction accuracy per individual participant. This provides a larger data set compared to deriving a MOS. However, this omits the desired noise reduction achieved by deriving a MOS and might introduce issues due to differences between participants. In fact, one major goal of applying the defined-use method is to derive a MOS and thus abstract from the individual participants towards an average observer. The prediction of individual judgments seems to be useful for verification of established models while it seems limited with regard to creating models.

\section{Subjective experiments}

In the following, the two experiments are presented that were conducted to investigate the formation process of multi-episodic perceived quality in one session. These extend the work of Guse et al. [12]. Following presented prior work, the defined-use method was applied. Speech telephony was selected as a service, because it is well investigated with regard to perceived quality. The two experiments follow a similar experimental design (i.e., hypotheses, performance levels, and multi-episodic conditions). As the impact of the usage situation on episodic judgments and multi-episodic judgments has not been investigated so far, the usage situation is purposefully varied. In Experiment 1 (E1), a two-party conversation was selected whereas in Experiment 2 (E2), a 3rd-party listening task was applied. ${ }^{6}$ Similar to prior work, only two performance levels were applied, which are shared between E1 and E2. High Performance (HP) denotes the highest performance, yielding only very limited to no perceptible degradations. Low Performance (LP) denotes the worst performance and is expected to provide a severely lower perceived quality. For LP, task solvability is necessary to avoid an impact of the potential arising frustration, which might undesirable affect judgments of perceived quality. In the following, the experimental design is presented.

\section{Hypotheses}

In the two experiments, the following hypotheses with regard to the formation process of multi-episodic perceived quality are investigated.

\footnotetext{
6 The two here presented experiments are included in the dissertation of Guse [9]. In difference to Guse, E2a is denoted in this paper as E2.
}

H1: Number of LP Usage Episodes Increasing the number of LP usage episodes before a multi-episodic judgment decreases this judgment.

This complements the results of Guse et al. [12]. They observed a duration neglect for the duration of one LP usage episode. However, their work leaves open if increasing the number of LP usage episodes, thus increasing the overall experienced duration of LP, also yields a duration neglect. This hypothesis is investigated in E1 and E2.

H2: Impact of Position of LP Usage Episodes The more HP usage episodes are presented directly before a multiepisodic judgment, the lower is the negative impact of LP usage episodes presented earlier.

Here, it is assumed that a recency effect occurs for multi-episodic judgments. In fact, a recency effect on multi-episodic judgments was indicated in prior work, but could not be proven conclusively (e.g., [21, 31]). This hypothesis is investigated in E1 and E2.

H3: Non-Consecutive vs. Consecutive LP Usage Episodes Presenting LP usage episodes non-consecutively (i.e., presenting HP usage episodes in between) leads to a higher reduction in multi-episodic judgments than presenting the same number of LP usage episodes consecutively.

It is expected that the higher number of changes in performance between usage episodes is undesirable as the service seems to be more unreliable. This hypothesis is investigated in E2 only.

The impact of the usage situation on multi-episodic perceived quality will be analyzed based upon the multiepisodic conditions investigating $\mathrm{H} 1$ and $\mathrm{H} 2$.

\section{Multi-episodic conditions, judgments, and performance levels}

For the investigation of the hypotheses, seven multi-episodic conditions were created. Per multi-episodic condition, we chose to employ six usage episodes that were presented sequentially in one session. This limits the duration of the multi-episodic condition to at most $45 \mathrm{~min}$. This avoids a potential impact of fatigue and limits the effort required by participants (cf. [44]). The number of usage episodes per multi-episodic condition is also similar to the experiment of Guse et al. [12].

In line with prior work, for all multi-episodic conditions the first three usage episodes were presented in HP (i.e., a well-working service is presented initially). LP might be presented only for usage episodes 4-6. After finishing each usage episode, the episodic perceived quality is assessed on the continuous 7-point scale [19] shown in Fig. 1. ${ }^{7}$ These episodic judgments allow to assess the impact of the

\footnotetext{
7 The continuous 7-point scale [19] was used also in presented prior work (cf. "Prior work on multi-episodic perceived quality").
} 


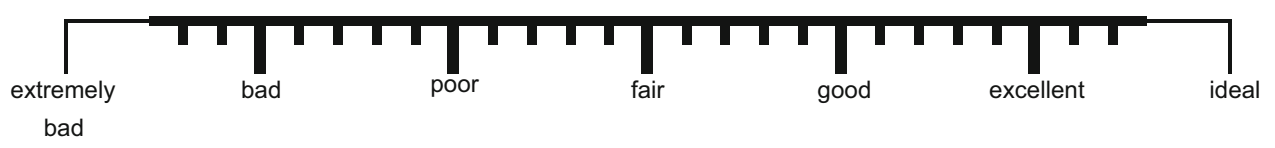

Fig. 1 Continuous 7-point scale (labels in English); used German labels from left-to-right: extrem schlecht (0), schlecht (1), dürftig (2), ordentlich (3), gut (4), ausgezeichnet (5), and ideal (6) [19]

presented performance levels on the perceived quality. For multi-episodic judgments, the same scale is presented asking for the perceived quality of all so far experienced usage episodes. ${ }^{8}$ Multi-episodic judgments are taken after the 3rd and 6th usage episode. Comparing the episodic judgments as well as the multi-episodic judgment after the 3rd usage episode between the multi-episodic conditions allows to investigate the impact of the applied betweensubject design.

An overview of the seven multi-episodic conditions and their corresponding hypotheses is given in Table 2. H1, H2, and $\mathrm{H} 3$ will be evaluated by comparing the multi-episodic judgment after the 6th usage episode (i.e., after the presentation of LP usage episode(s)). For H1, the multi-episodic conditions $\mathrm{C} 3, \mathrm{C} 5$, and $\mathrm{C} 6$ are compared. These present consecutively one, two, or three LP usage episodes directly before this multi-episodic judgment. Additionally, $\mathrm{C} 2$ and $\mathrm{C} 4$ are compared, which present the 6th usage episode in HP while presenting one or two LP usage episodes beforehand. H2 (i.e., the impact of position of LP usage episode(s) towards the following multi-episodic judgment) is evaluated by comparing $\mathrm{C} 1, \mathrm{C} 2$, and $\mathrm{C} 3$ as well as $\mathrm{C} 4$ and $\mathrm{C} 5$. These present one or two LP usage episodes followed by none, one, or two HP usage episodes before the following multi-episodic judgment. H3 is evaluated by comparing $\mathrm{C} 7$ with $\mathrm{C} 4$ and $\mathrm{C} 5$. The multi-episodic condition that presents all usage episodes in HP is omitted, as it is assumed that the multi-episodic judgments remain on the same level. In fact, the potential increase observed by Möller et al. [29] was not significant and, if existing at all, is rather small (approximately $0.3 \mathrm{pt}$ on the continuous 7-point scale). As approximation for this multiepisodic condition, the multi-episodic judgment after the 3rd usage episode is used. This judgment is in the following denoted reference.

Performance levels were selected to provide nearly constant degradations. This should avoid undesired effects of varying perceived quality within a usage episode as potential effects are not yet fully understood. This was achieved by varying the speech codec between HP and LP. Furthermore, the performance levels were required to be

\footnotetext{
${ }^{8}$ In the following, the term judgment is used in singular if referring to measurements after the same (in terms of time) usage episode. This is even applied if describing different multi-episodic conditions. Plural is used if the judgments were taken after different (in terms of time) usage episodes.
}

noticeably different. For HP, G.722 was selected as this is a reference standard for wideband speech transmission. For LP, LPC-10 was selected. LPC-10 was, in fact, not designed for speech telephony but instead for low bandwidth speech transmission via radio. It provides intelligibility while the re-synthesized speech signal sounds very unnatural. Selecting LPC-10 enables to create a nearly constant severe degradation while the service remains sufficiently usable to solve conversational tasks. On the one hand, using LPC-10 might limit generalizability of experimental results, as it is not used for speech telephony. On the other hand, it is a severe degradation that is reproducible and is independent of the current state of technology. Therefore, LPC-10 can be used as baseline for future investigations on multi-episodic perceived quality.

To the knowledge of the authors, LPC-10 was not evaluated with regard to perceived quality. Therefore, we computed the MOS using POLQA [20] (super-wideband mode) with $12 \mathrm{~s}$ German speech samples. The results are shown in Table 3. This evaluation showed that LPC-10 provides only a very low MOS, which is very different to G.722.

\section{Tasks}

For E1, two-party conversation was desired as this is the common usage situation for speech telephony. Here, the SCSs [18] were used. In these standardized scenarios one participant takes the role of the caller, who needs to achieve his goal by informing the callee about his needs. Based upon the provided information the callee presents a suiting solution to the caller, and a final information transfer is conducted. While SCSs define the information to be exchanged and a conversational structure is suggested, the actual behavior of caller and callee is not limited. Thus, every conversation will be different in terms of timing, number of speaker changes etc. SCSs are in general solved in 2-3 min.

In E2, 3rd-party listening was used to avoid an impact of varying user behavior. Here, a participant takes the role of a passive listener in a multi-party conversation with two active speakers. It was chosen to use recordings of SCSbased conversations acquired in E1. The duration of these recordings ranged from 128 to $184 \mathrm{~s}$ with male and female speakers. As task, six questions, which resemble the implicit questions applied in the SCSs, had to be answered 
Table 2 Overview of all multiepisodic conditions (incl. performance level per usage episode) and which are compared for the evaluation of the three hypotheses

\begin{tabular}{|c|c|c|c|c|c|c|c|c|c|c|}
\hline \multirow[t]{2}{*}{ Condition } & \multicolumn{6}{|c|}{ Episodic performance level } & \multicolumn{3}{|c|}{ Hypotheses } & \multirow[t]{2}{*}{ Experiment } \\
\hline & 1 & 2 & 3 & 4 & 5 & 6 & H1 & $\mathrm{H} 2$ & $\mathrm{H} 3$ & \\
\hline $\mathrm{C} 1$ & HP & HP & HP & LP & HP & HP & - & * & - & E1, E2 \\
\hline $\mathrm{C} 2$ & HP & HP & HP & HP & $\mathbf{L P}$ & $\mathrm{HP}$ & $*$ & $*$ & - & E1, E2 \\
\hline C3 & HP & HP & HP & HP & HP & LP & $\star$ & $*$ & - & E1, E2 \\
\hline $\mathrm{C} 4$ & HP & HP & HP & LP & LP & HP & $*$ & $\star$ & - & E1, E2 \\
\hline C5 & HP & HP & HP & HP & LP & LP & $\star$ & $\star$ & $*$ & E2 \\
\hline C6 & HP & HP & HP & LP & LP & LP & $\star$ & - & $*$ & E1, E2 \\
\hline C7 & HP & HP & HP & LP & HP & LP & - & - & $*$ & E2 \\
\hline
\end{tabular}

LP usage episodes are in bold. Position of the multi-episodic judgments are marked by double vertical lines
Table 3 Comparison of the G.722 (HP) and LPC-10 (LP) using POLQA

\begin{tabular}{llll}
\hline Performance & Signal bandwidth & Codec & POLQA \\
\hline HP & $50 \ldots 7000 \mathrm{~Hz}$ & G.722, Mode 1 & 4.0 \\
LP & $300 \ldots 3400 \mathrm{~Hz}$ & LPC-10 & 1.9 \\
\hline
\end{tabular}

The prediction was transformed to the 7-point continuous scale by applying the mapping presented by Köster et al. [26]

(e.g., "What is the name of the caller?" or "What is the caller requesting?"). This should ensure that participants follow attentively the content of the conversation.

\section{Procedure and systems}

In both experiments, a very similar procedure was applied consisting of two parts. In Part I, one listening-only quality assessment of short speech stimuli was conducted. This ensures that all participants experience the same telephonyrelated impairments. It is assumed that this quality assessment provides participants with a shared reference for the following assessment of the multi-episodic condition. We opted to present a broad range of typical telephony-related importants. This avoids priming participants only to the two impairments to be presented in the multi-episodic conditions. The impairments included different signal bandwidths (narrowband, wideband), codecs (G.711, G.722, GSM-FR, LPC-10), white-noise (fullband), and random packet loss (2 and 5\%; G.722 Mode 1, concealment: zero insertion). Overall, the perceived quality of 27 stimuli of $8 \mathrm{~s}$ duration was evaluated on the 7-point continuous scale (cf. Fig. 1). In Part II, one multi-episodic condition was presented and assessed. In case of E1, two participants conducted this part together assessing the same multi-episodic condition. In case of E2, this part was conducted by each participant alone.

In E1, Part I was conducted on a tablet computer $(F u$ jitsu Stylistic ST6012). For diotic representation of the stimuli, a pair of $A K G K-271$ headphones was connected to the internal sound card of the tablet computer. The system was calibrated to a sound pressure level of $75 \mathrm{~dB}$ SPL for babble noise using a HEAD acoustics head and torso simulator HSM II.3. In Part II, the two participants were placed into separate rooms fulfilling ITU-T Recommendation P.800 [17]. For each of the two participants, audio capturing and diotic representation were provided using a Beyerdynamic DT 790 Pro headset. The headsets were connected to an Edirol UA25-EX sound card. The used transmission system provided a constant, glitch-free oneway end-to-end delay below $120 \mathrm{~ms}$.

In E2, Part I and Part II were conducted using one pair of Sennheiser HD 25-1 headphones connected to the internal sound card of a Microsoft Surface Pro (1st generation). The sound pressure level was again calibrated to $75 \mathrm{~dB}$ SPL for babble noise. This experiment was also conducted in a room fulfilling ITU-T Recommendation P.800 [17].

\section{Participants}

The two experiments were conducted in Berlin, Germany from April 2014 to December 2015. E1 was conducted with 51 female and 35 male participants aged from 18 to 53 years $(\mu=27.2, \sigma=6.8)$. In this experiment, participants did not know each other beforehand. E2 was conducted with 75 female and 40 male participants aged from 18 to 50 years $(\mu=26.2, \sigma=4.6)$. Participants of E1 were excluded from E2. A between-subject design was applied (i.e., only one multi-episodic condition was presented to each participant). The number of participants per multiepisodic condition is shown in Table 4.

Table 4 Number of participants by multi-episodic condition and experiment

\begin{tabular}{llllllll}
\hline Experiment & \multicolumn{7}{l}{ Multi-episodic condition } \\
\cline { 2 - 7 } & C1 & C2 & C3 & C4 & C5 & C6 & C7 \\
\hline E1 & 18 & 15 & 13 & 11 & 16 & 13 & - \\
E2 & 12 & 15 & 13 & 15 & 24 & 21 & 15 \\
\hline
\end{tabular}


For both experiments, participants were assigned randomly to one multi-episodic condition. In case of E1, each pair of participants was assigned to the same multi-episodic condition.

\section{Data analysis}

In the following, the results of both experiments are presented. First, episodic judgments are inspected with regard to a potential undesired impact of the applied betweensubject design. Second, the final multi-episodic judgment is evaluated with regard to the presented hypotheses. In addition, the impact of the usage situation (i.e., differences between experiments) is investigated using episodic judgments and multi-episodic judgments.

All results are reported as MOS ranging from 0 to 6 (for the scale cf. Fig. 1) with standard deviation in brackets. For the statistical evaluation of two unpaired samples, a Wilcoxon rank-sum test is conducted. More than two unpaired samples are compared by conducting a Kruskal-Wallis test and, if significant differences are found, a pairwise Wilcoxon rank-sum test with Holms' correction is administered. A significance level of $\alpha=0.05$ is applied. If two groups are significantly different, the Hodges Lehmann estimator $\hat{\triangle}$ is reported.

\section{Episodic judgments}

For both experiments, each multi-episodic condition was individually checked for inconsistent judging participants. The judgments of a participant are considered inconsistent if more than two episodic judgments are beyond $1.5 \times$ interquartile range. This conservative criterion was chosen as no prior knowledge about typical characteristics of inconsistent judgments with regard to multi-episodic evaluation was available. This criterion was not met by any participant, and thus none was excluded from the data analysis. In prior work, a negative influence on the episodic judgments of HP usage episodes was observed if these follow directly LP usage episode(s) (cf. [6, 29]). A detailed investigation did not show the presence of such an effect for any of the multi-episodic conditions evaluated in E1 and E2.

With regard to consistency between multi-episodic conditions, E1 shows no significant difference of episodic judgments for HP usage episodes $(H(5)=10.8681$, $p=0.0541)$. For E2, a significant difference between the episodic judgments of HP usage episodes is found $(H(6)=13.9445, p=0.0303)$. However, conducting a post-hoc test did not show significant differences between the multi-episodic conditions $(p \geq 0.066)$. A detailed analysis did not yield a reason for these differences, and we therefore consider this an artifact due to the between-subject design not affecting the hypotheses testing. For LP, significant differences of episodic judgments are neither observed in E1 $(H(5)=5.789, p=0.3273)$ nor E2 $(H(6)=4.317, p=0.6339)$. For both experiments, we thus conclude that the results are consistent with regard to episodic judgments.

Between HP and LP, significant differences are found per experiment $(\mathrm{E} 1: W=58377.0, p<0.001, \hat{\triangle}=2.8$ and $\mathrm{E} 2: W=98931.0, p<0.001, \hat{\triangle}=2.5)$. For HP, E1 yielded $4.1(0.7)$, and E2 yielded $4.2(0.8)$. For LP, E1 yielded 1.5 (0.7), and E1 yielded 1.8 (0.8). Between the two experiments, episodic judgments of HP are not significantly different $(W=94592.0, p=0.065)$. With regard to LP, both experiments show a significant difference $(W=10800.0$, $p<0.001, \hat{\triangle}=-0.3)$. This indicates that the presentation of usage episodes in LP is judged worse in a conversational usage situation than in a listening-only usage situation for the selected performance level (i.e., LPC-10). Thus, the usage situation seems to influence the formation process of episodic judgments although the effect size is rather small. To give an exemplary illustration of episodic judgments, Fig. 2 shows a boxplot for C6 (E1).

\section{Multi-episodic judgments}

In the following, the results of the multi-episodic judgments are evaluated. First, consistency between the multiepisodic conditions is analyzed with regard to the reference (i.e., the multi-episodic judgment after the 3rd usage episode). Subsequently, the results are evaluated in detail with regard to the hypotheses under investigation. With regard to this multi-episodic judgment, no significant difference between the multi-episodic conditions is found per experiment $(\mathrm{E} 1: \quad H(5)=2.0883, \quad p=0.8368$ and $\mathrm{E} 2$ : $H(6)=6.7373, p=0.3458)$. Also, the two experiments do not differ significantly $(H(7)=4.729, p=0.693$; E1: 4.3 (0.6), E2: $4.3(0.8))$. This indicates that, as long as only HP usage episodes were presented, neither the between-subject design nor the difference in usage situation seemed to affect this multi-episodic judgment. Figure 3 and Table 5 show the multi-episodic judgments per multi-episodic condition for the two experiments.

\section{Hypothesis H1: number of LP usage episodes}

In $\mathrm{H} 1$, it is assumed that increasing the number of LP usage episodes before a multi-episodic judgment results in a decrease of this judgment. This hypothesis is evaluated by comparing the reference with the multi-episodic judgment 
Fig. 2 Boxplot for episodic judgments of C6 (E1). The 4th, 5 th, and 6th usage episode were presented in LP

Fig. 3 Boxplot of multiepisodic judgments per multiepisodic condition and experiment for E1 and E2
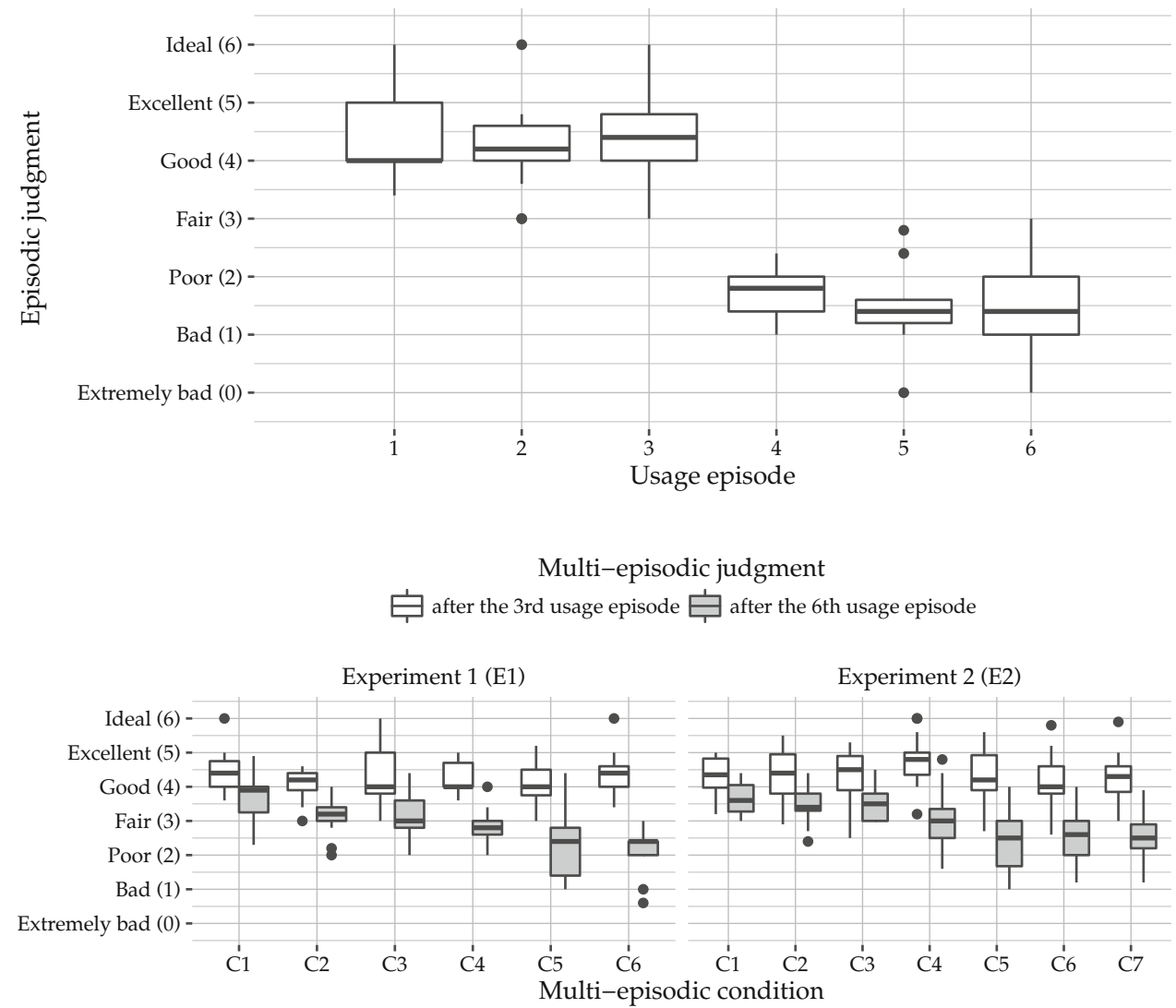

Multi-episodic judgment

Multi-episodic condition
Table 5 Multi-episodic judgments per multi-episodic condition and experiment for E1 and E2

\begin{tabular}{|c|c|c|c|c|}
\hline \multirow[t]{3}{*}{ Condition } & \multicolumn{4}{|c|}{ Multi-episodic judgment } \\
\hline & \multicolumn{2}{|l|}{ E1 } & \multicolumn{2}{|l|}{ E2 } \\
\hline & 3rd episode & 6th episode & 3rd episode & 6th episode \\
\hline $\mathrm{C} 1$ & $4.4(0.6)$ & $3.7(0.6)$ & $4.3(0.6)$ & $3.6(0.5)$ \\
\hline $\mathrm{C} 2$ & $4.1(0.4)$ & $3.2(0.6)$ & $4.4(0.7)$ & $3.5(0.5)$ \\
\hline $\mathrm{C} 3$ & $4.3(0.8)$ & $3.1(0.8)$ & $4.3(0.8)$ & $3.5(0.5)$ \\
\hline $\mathrm{C} 4$ & $4.2(0.5)$ & $2.9(0.5)$ & $4.8(0.7)$ & $3.0(0.9)$ \\
\hline C5 & $4.1(0.6)$ & $2.3(0.9)$ & $4.2(0.8)$ & $2.4(0.9)$ \\
\hline C6 & $4.3(0.7)$ & $2.1(0.7)$ & $4.1(0.8)$ & $2.5(0.8)$ \\
\hline $\mathrm{C} 7$ & _- & - & $4.3(0.8)$ & $2.5(0.6)$ \\
\hline
\end{tabular}

after the 6th usage episode of $\mathrm{C} 3, \mathrm{C} 5$, and $\mathrm{C} 6$ as well as $\mathrm{C} 2$ and $\mathrm{C} 4 . \mathrm{C} 3, \mathrm{C} 5$, and $\mathrm{C} 6$, present one to three LP usage episodes before the final multi-episodic judgment. $\mathrm{C} 2$ and $\mathrm{C} 4$, present one or two LP usage episodes followed by one HP usage episode before the final multi-episodic judgment.

For these five multi-episodic conditions, Table 6 shows the final multi-episodic judgment as well as the statistical evaluation. For E1 and E2, the results show that increasing the number of LP usage episodes from zero (i.e., the reference) to one as well as from one to two leads to a decrease in this multi-episodic judgment. Presenting a third usage episode in LP did not result in a further decrease. In fact, this multi-episodic judgment remained above the episodic judgments of LP usage episodes (i.e., the difference is approximately $1 \mathrm{pt}$ ). This indicates that priorly presented HP usage episodes still attribute to this multi-episodic judgment. This observation is in the following denoted as saturation. Notably, E1 and E2 show very similar results with regard to the multi-episodic conditions. Nevertheless, it must be noted that in absolute numbers the final multi-episodic judgment seems to be higher for E2 than for E1. This observation might be attributable to the different usage situations.

Hypothesis H2: impact of the position of LP usage episodes

In $\mathrm{H} 2$, it is hypothesized that presenting HP usage episodes after presenting one or more LP usage episode(s) reduces the negative impact on the following multi-episodic judgment. This was investigated for one and two LP usage episodes with regard to the final multi-episodic judgment in $\mathrm{E} 1$ and $\mathrm{E} 2 . \mathrm{C} 1, \mathrm{C} 2$, and $\mathrm{C} 3$ present each one LP usage episode with either two, one, or no following HP usage episode(s) before this judgment. C4 and C5 present two 
Table 6 (Hypothesis 3) Comparison of the reference and the multi-episodic judgment after the 6th usage episode for $\mathrm{E} 1$ and E2

\begin{tabular}{|c|c|c|c|c|c|c|}
\hline & \multicolumn{3}{|l|}{ E1 } & \multicolumn{3}{|l|}{ E2 } \\
\hline & \multicolumn{3}{|c|}{ Reference: 4.3 (0.6) } & \multicolumn{3}{|c|}{ Reference: $4.3(0.8)$} \\
\hline & \multicolumn{3}{|c|}{$H(3)=53.2096, p<0.001$} & \multicolumn{3}{|c|}{$H(3)=65.084, p<0.001$} \\
\hline & $\begin{array}{l}\text { C3 } \\
3.1(0.8)\end{array}$ & $\begin{array}{l}\text { C5 } \\
\mathbf{2 . 3}(\mathbf{0 . 9})\end{array}$ & $\begin{array}{l}\text { C6 } \\
2.1(0.7)\end{array}$ & $\begin{array}{l}\text { C3 } \\
3.5(\mathbf{0 . 5})\end{array}$ & $\begin{array}{l}\text { C5 } \\
\mathbf{2 . 4}(\mathbf{0 . 9})\end{array}$ & $\begin{array}{l}\text { C6 } \\
\mathbf{2 . 5}(\mathbf{0 . 8})\end{array}$ \\
\hline Reference & $\begin{array}{l}W=71.0, \\
p<0.001, \\
\hat{\triangle}=-1.0\end{array}$ & $\begin{array}{l}W=31.0 \\
p<0.001 \\
\hat{\triangle}=-2.0\end{array}$ & $\begin{array}{l}W=1.0 \\
p<0.001 \\
\hat{\triangle}=-2.0\end{array}$ & $\begin{array}{c}W=169.0 \\
p=0.002 \\
\hat{\Delta}=-0.8\end{array}$ & $\begin{array}{l}W=81.5 \\
p<0.001 \\
\hat{\triangle}=-1.8\end{array}$ & $\begin{array}{l}W=76.0 \\
p<0.001 \\
\hat{\Delta}=-1.7\end{array}$ \\
\hline $\mathrm{C} 3$ & - & $\begin{array}{l}W=49.0 \\
p=0.016, \\
\hat{\Delta}=-0.8\end{array}$ & $\begin{array}{c}W=31.0, \\
p=0.009, \\
\hat{\triangle}=-1.0\end{array}$ & - & $\begin{array}{l}W=40.5 \\
p<0.001 \\
\hat{\triangle}=-1.2\end{array}$ & $\begin{array}{l}W=31.0 \\
p<0.001 \\
\hat{\Delta}=-1.0\end{array}$ \\
\hline C5 & - & - & $\begin{array}{l}W=89.0 \\
p=0.261\end{array}$ & - & - & $\begin{array}{l}W=278.0 \\
p=0.727\end{array}$ \\
\hline
\end{tabular}

A one-sided post-hoc test is applied. MOS printed bold with standard deviation in brackets
Table 7 (Hypothesis 2) The multi-episodic judgment after the 6th usage episode for E1 and E2

\begin{tabular}{llll}
\hline Condition & LP usage episode(s) & E1 & E2 \\
\hline C1 & 4 & $3.7(0.6)$ & $3.6(0.5)$ \\
C2 & 5 & $3.2(0.6)$ & $3.5(0.5)$ \\
C3 & 6 & $3.1(0.8)$ & $3.5(0.5)$ \\
C4 & $4-5$ & $2.9(0.5)$ & $3.0(0.9)$ \\
C5 & $5-6$ & $2.3(0.9)$ & $2.4(0.9)$ \\
\hline
\end{tabular}

Reported as MOS with standard deviation in brackets

consecutive LP usage episodes while one or no HP usage episode are presented directly before this judgment. Table 7 shows the multi-episodic judgment after the 6th usage episode for these multi-episodic conditions.

For E1, this multi-episodic judgment is significantly different between $\mathrm{C} 1, \mathrm{C} 2$, and $\mathrm{C} 3(H(2)=7.0608$, $p=0.0293)$. A one-sided post-hoc test shows that $\mathrm{C} 1$ is significantly different from $\mathrm{C} 2(W=72.0, p=0.032$, $\hat{\Delta}=-0.6)$ and $\mathrm{C} 3(W=63.5, p=0.032, \hat{\Delta}=-0.4)$, but $\mathrm{C} 2$ and $\mathrm{C} 3$ are not significantly different $(W=94.0$, $p=0.444) . \quad \mathrm{C} 4$ and $\mathrm{C} 5$ are significantly different ( $W=126.0, p=0.031, \hat{\Delta}=0.6$, one-sided). For E2, C1, $\mathrm{C} 2$, and $\mathrm{C} 3$ are not significantly different $(H(2)=0.6447$, $p=0.7245)$. In contrast, $\mathrm{C} 4$ and $\mathrm{C} 5$ are significantly different $(W=249.5, p=0.023, \hat{\Delta}=0.6$, one-sided).

With regard to $\mathrm{H} 2$, both experiments yield similarities and differences. For E1, presenting LP usage episode(s) earlier reduced the negative impact on the following multi-episodic judgment. It must be noted that this could be observed for one LP usage episode only if more than one HP usage episode was presented subsequently. For E2, an impact of position could not be observed for one
LP usage episode but for two LP usage episodes. It can thus be concluded that varying the position of LP usage episodes towards the following multi-episodic judgment affects this judgment only under certain conditions. This suggests that a recency effect could be observed in both experiments. However, the observed differences between the two experiments indicate an impact of the usage situation on the formation process of multi-episodic judgments. Thus, $\mathrm{H} 2$ can be accepted in case of two-party conversations (E1), but can only be partly accepted for 3rdparty listening (E2).

Hypothesis H3: non-consecutive vs. consecutive LP usage episodes

In H3, it is assumed that the presentation of consecutive LP usage episodes yields a higher multi-episodic judgment than the same number of LP usage episodes presented nonconsecutively (E2 only). This hypothesis is evaluated by comparing the multi-episodic judgment after the 6th usage episode of $\mathrm{C} 7$ with $\mathrm{C} 4$ as well as $\mathrm{C} 5$. C4 and C5 both present two LP usage episodes consecutively. In C7, the 4th and 6th usage episode are presented in LP. Table 8 shows the final multi-episodic judgment for these three multi-episodic conditions. Between these multi-episodic conditions, the final multi-episodic judgment is not

Table 8 (Hypothesis 3) The multi-episodic judgment after the 6th usage episode for E1 and E2

\begin{tabular}{lll}
\hline Condition & LP usage episode(s) & E2 \\
\hline C4 & $4-5$ & $3.0(0.9)$ \\
C7 & 4 and 6 & $2.5(0.6)$ \\
C5 & $5-6$ & $2.4(0.9)$ \\
\hline
\end{tabular}

Reported as MOS with standard deviation in brackets 
significantly different $(H(2)=4.3146, \quad p=0.1156)$. However, the multi-episodic judgment of $\mathrm{C} 7$ seems to be closer compared to $\mathrm{C} 5$ than to $\mathrm{C} 4$. This might indicate a higher impact of the last usage episode in terms of recency. Anyhow, H3 must be rejected given the results.

\section{Summary of findings}

The investigation of the three hypotheses yielded important insights into the formation process of multi-episodic perceived quality. Most notably, similarities and differences between two-party conversation (E1) and third-party listening (E2) were observed.

For H1 (i.e., impact of varying the position of LP usage episodes), both experiments yielded similar findings. Here, a decrease of the final multi-episodic judgment is observed if the number of LP usage episodes is increased from zero to two directly before this judgment. Although multi-episodic judgments remained above episodic judgments of LP usage episodes, presenting three LP usage episodes did not lead to a further decrease. It is thus concluded that three consecutive LP usage episodes are either judged differently or that the importance of HP usage episodes is assessed differently.

For H2 (i.e., varying the position of one or two consecutive LP usage episodes), the results of E1 showed consistently that presenting LP usage episodes earlier decreases the reduction of the following multi-episodic judgment. For E2, this could only be observed in case of two LP usage episodes, but not for one LP usage episode. The underlying reason for this difference between E1 and E2 could not be determined. This difference suggests that the multi-episodic formation process is affected by the usage situation.

For H3 (i.e., presenting LP usage episodes consecutively vs. non-consecutively), no significant effect could be observed (E2 only). The results suggest that presenting the last usage episode before the multi-episodic judgment in LP has a higher impact than the potential effect of presenting LP usage episodes non-consecutively. Therefore, we rejected $\mathrm{H} 3$ as either the effect is too small to be observed or does not exist at all.

\section{Prediction of the final multi-episodic judgment}

In the following, the multi-episodic judgment after the 6th usage episode is predicted. Möller et al. [29] and Guse et al. [11] evaluated predictors based upon judgments of individual participants. In difference to their approach, the evaluation of potential predictors is conducted here based upon the MOS alone (cf. end of section "Perceived quality for multi-episodic usage"). Especially, due to the sparse knowledge about the formation process of multi-episodic judgments, this seems to be the more suiting approach. In fact, the defined-use method was applied, so the noise reduction due to deriving the MOS can be utilized.

Guse et al. [11] proposed to compute the weighted average of prior episodic judgments to predict a multiepisodic judgment. This allows to assign a distinct weight to each episodic judgment and thus account for its impact on the multi-episodic judgment to be predict. For prediction, we follow this approach while using the MOS. In the following, episodic judgments are denoted as $e_{i}$ and multiepisodic judgments are denoted as $m_{n}$. The index $i$ denotes the usage episodes in their chronological order, and the index $n$ denotes the usage episode after which a multiepisodic judgment was taken. The weight for an episodic judgment is denoted as $a_{i}$. Thus, the prediction model $\hat{m}_{n}$ is defined as:

$$
\hat{m}_{n}=\frac{\sum_{i=1}^{n} a_{i} \cdot e_{i}}{\sum_{i=1}^{n} a_{i}} .
$$

The weighted average is a rather simple model type as it is only parametrized by a weight function. Following Guse et al. [11], a window function (WI) and a linear weight function (LI) are proposed (cf., Eqs. 2 and 3).

$$
\begin{aligned}
& W I: a_{i}=\left\{\begin{array}{cc}
1, & \text { if } \mathrm{i}-\mathrm{n}+\mathrm{w}>0 \\
0, & \text { otherwise }
\end{array}\right. \\
& L I: a_{i}=\left\{\begin{array}{cc}
i-n+w, & \text { if } \mathrm{i}-\mathrm{n}+2 \cdot \mathrm{w}>0 \\
0, & \text { otherwise }
\end{array}\right.
\end{aligned}
$$

Both weight functions are parametrized by $w$, which denotes the window size to be applied. $w$ is limited to $w \in \mathbb{N}$ and $0<w \leq n$. Please note that the effective window size of LI is $2 \cdot w$ (cf. Eq. 3). Otherwise, the episodic judgment of the very first usage episode would always be weighted with $a_{1}: 0$. The prediction accuracy is evaluated using the Root Mean Squared Deviation (RMSD). A prediction model (i.e., the weight function incl. its manifestation of $w$ ) is preferable that explains a higher number of multi-episodic conditions rather than specific multi-episodic conditions alone. The prediction accuracy is evaluated for the multi-episodic judgment after the 6th usage episode.

The RMSD depending on $w$ is shown in Fig. 4 for E1 and Fig. 5 for E2. Comparing the results per weight function shows that the overall prediction accuracy behaves similarly for both experiments. For WI, the RMSD decreases until $w=4$. However, the RMSD depends on the considered multi-episodic condition. For example, C3 is far off for $w=1$ and improves until $w=3$ while $\mathrm{C} 4$ is best predicted by $w=5$. For WI, the minimal RMSD is reached at $w=4$ (E1: 0.22 and E2: 0.18$)$ while $w=3$ is rather close. LI outperforms WI with regard to the RMSD and is, furthermore, more robust. LI achieves the lowest RMSD at 
Fig. 4 RMSD for the multiepisodic MOS after the 6th usage episode for all multiepisodic conditions of E1

Fig. 5 RMSD for the multiepisodic MOS after the 6th usage episode for all multiepisodic conditions of E2
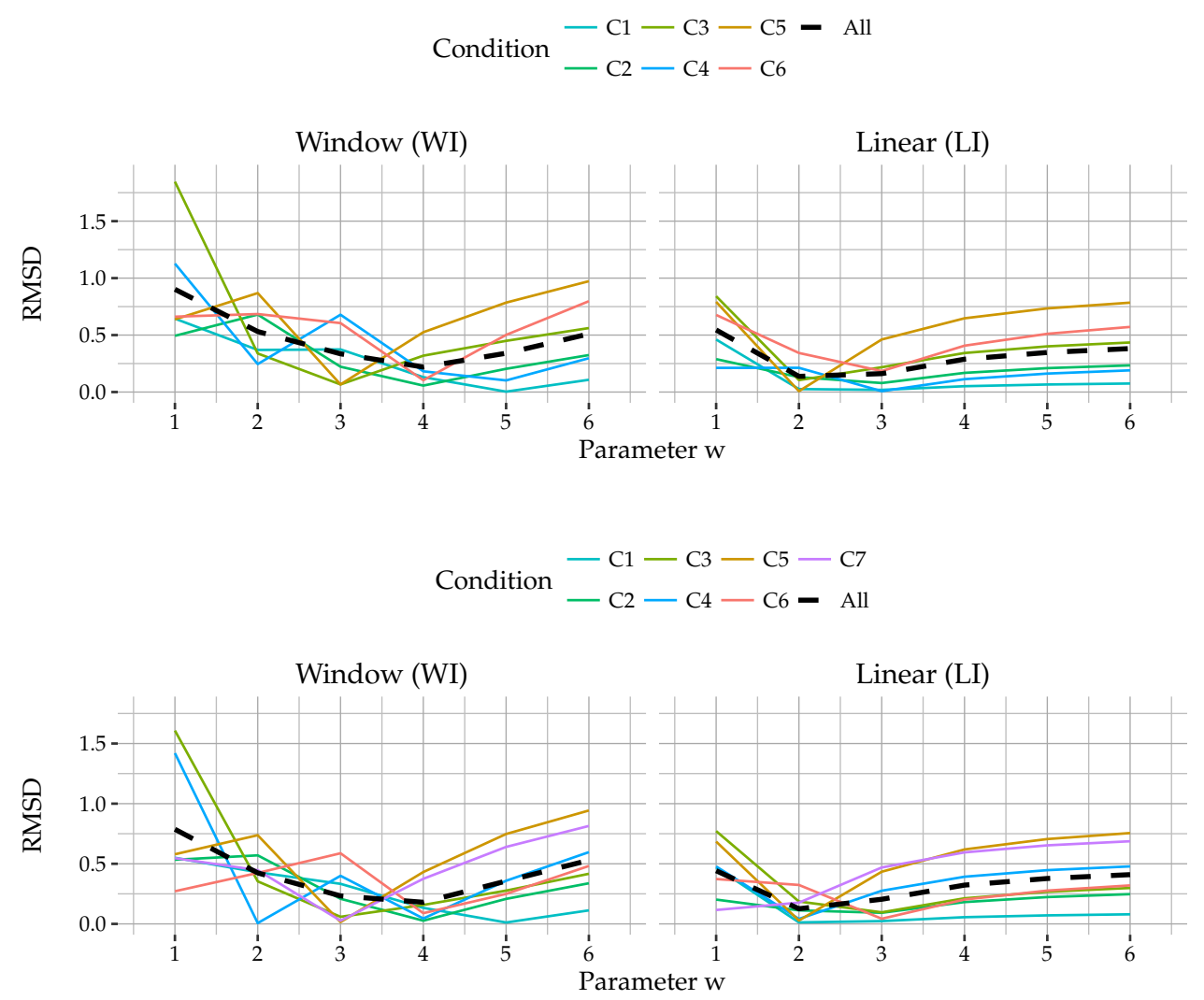

$w=2$ (E1: 0.14 and E2: 0.13). Here, all multi-episodic conditions except $\mathrm{C} 4$ and $\mathrm{C} 6$ yield their minimal RMSD. C4 and C6 reach their lowest RMSD at $w=3$.

The evaluation shows that both weight functions can be used for predicting the multi-episodic judgment after the 6th usage episode using episodic judgments (both in terms of MOS). Both weight functions achieve the lowest RMSD and thus best prediction accuracy if the four most recent usage episodes are considered (i.e., WI: $w \leq 4$ and LI: $w \leq 2$ ).

With regard to the saturation that was observed in E1 and E2, the application of the proposed weighted average is necessarily limited. In both experiments, it was observed that C5 and C6 resulted in a non-different final multi-episodic judgment although these two multi-episodic conditions differ in the performance level of the 4th usage episode. In case of C5, this usage episode was presented in HP and in case of C6 in LP. For both multi-episodic conditions, the 5th and 6th usage episode were presented in LP. The multi-episodic judgment remained approximately $1 \mathrm{pt}$ higher than the episodic judgment of LP usage episodes. With regard to the two weight functions, this is an issue as both would need to produce the same output using different inputs. This implies a window size of less than 2 , but for both weight functions the lowest RMSD was found at an effective window size of 4 .

Overall, we conclude that a weighted average can be used to predict the multi-episodic judgment after the 6 th usage episode of the here presented experiments using episodic judgments.

\section{Conclusion, discussion, and future work}

In the two presented experiments, the formation process of multi-episodic perceived quality in one session (up to $45 \mathrm{~min}$ ) was investigated. Here, a session consists of multiple, similar usage episodes with the same service while usage episodes are self-contained, task-driven interactions. For this investigation, the defined-use method was applied, which allows to derive a MOS as the same multiepisodic condition can be presented to multiple participants. By varying only the performance level of specific usage episodes between multi-episodic conditions, the impact of these differences on the formation process of multi-episodic judgments can be investigated. Furthermore, applying the defined-use method has the advantage that experiments are repeatable (i.e., multi-episodic conditions incl. performance are reproducible).

With regard to multi-episodic perceived quality, three hypotheses were investigated. Here, the impact on the final multi-episodic judgment was studied for an increasing number of severely degraded usage episodes (H1), varying the position of severely degraded usage episodes $(\mathrm{H} 2)$, and the consecutive vs. non-consecutive presentation (H3). In 
addition, a potential impact of the usage situation (i.e., conversation vs. passive listening) was investigated.

Although the usage situation was varied, the observed findings were similar to a certain degree. In both experiments, the multi-episodic judgment decreased if more degraded usage episodes were presented until saturation was reached. Here, the multi-episodic judgment remained on the same level regardless whether two or three severely degraded usage episodes were presented. In fact, saturation occurred before reaching the level of the episodic judgments for severely degraded usage episodes. This indicates that the additionally presented severely degraded usage episode was not considered in the formation process of the multi-episodic judgment or that the weighting of the individual usage episodes on the multi-episodic judgment was different. However, the reason for this observation could not be determined. In addition, the impact of varying the position of severely degraded usage episodes was investigated in both experiments. Here, a recency effect could be observed in some cases for both usage situations. The results indicate that 3rd-party listening compared to twoparty conversation is less prone to show a recency effect, as it could only be observed for two usage episodes. Again, the underlying reason could not be determined, but it might be that two-party conversation is more mentally demanding. Finally, the non-consecutive vs. consecutive presentation of severely degraded usage episodes did not show a significant effect. Based upon the empirical results, prediction models in terms of curve fitting were created. Here, the weighted average using either a window function or a linear weight function was found to be sufficiently precise considering the extent of the data set and the applied between-subject design.

The results and findings are in itself consistent and are in line with prior work on the formation process of multiepisodic perceived quality. Nevertheless, they are inherently limited due to the experimental design. This includes the application of the defined-use method, the selected service type, and the applied performance levels. With regard to generalizability of the findings, it is not yet understood how these factors may affect the formation process of multi-episodic judgments. Especially, the selected performance levels seem to be of outstanding importance. For the presented experiments, LPC-10 was selected to create severely degraded usage episodes, as this speech codec provides only robotic but intelligible speech. This resulted, as desired, in a severe degradation, and the impact on the final multi-episodic judgment could be observed. It must be noted that LPC-10 is actually outdated and has never been used for speech telephony. Therefore, the resulting artifacts are not realistic. Nevertheless, selecting LPC-10 has the advantage that it can be easily recreated in the future as its implementation is of limited complexity. In addition to the selected performance levels, generalizing the findings is limited as the impact of potential influence factors in terms of Reiter et al. [33] has not yet been investigated. Although we believe that the observed effects are likely to occur, the actual effect sizes might be different.

Future work is necessary to understand the formation process of multi-episodic perceived quality. Here, the presented definition of usage episode that is derived from psychological concepts forms a starting point. It is important to investigate whether the findings can be generalized. This includes other types of telecommunication services, such as video transmission and web browsing, and also include repeated-use of software, such as gaming, in general. Depending on the service type, usage situation, context, and tasks, differences in effects on multi-episodic judgments might be observable. Beside performance, also macroscopic fluctuations leave room for future research (i.e., perceptible variations in performance within a usage episode). From a methodological perspective, it is important to evaluate the defined-use method in detail. This includes the impact of taking episodic judgments, which might affect the memorization of perceived quality and thus multi-episodic judgments. Also, the required forced usage is an issue to be investigated, as participants need to solve the provided task(s) while not necessarily being intrinsically motivated. Therefore, failure to solve a task might be perceived, memorized, and judged differently depending on the motivation. Here, also the importance of specific usage episodes should be considered for further investigation. In addition, the results obtained with the defined-use method need to be verified in terms of ecological validity. Furthermore, deriving a MOS assumes that there exists an average formation process of multi-episodic perceived quality. This necessarily neglects differences between individuals. Here, it is important to conduct research on potential individual differences in this formation process. We think that investigations of multi-episodic perceived quality should be primarily conducted with experiments especially designed for this purpose rather than being a byproduct.

A deeper understanding of multi-episodic perceived quality will be beneficial with regard to user behavior and its adaption. So far, user behavior has been excluded to be able to establish a baseline. Taking user behavior into account enables to determine the actual impact of varying service performance. This includes how the services is used, frequency of usage, and churn as well as customer behavior in general. Therefore, developing objective prediction models for multi-episodic perceived quality will enable service providers to improve their services. 


\section{References}

1. Ariely D (1998) Combining experiences over time: the effects of duration, intensity changes and on-line measurements on retrospective pain evaluations. J Behav Decis Making 11(1):19-45

2. Belmudez B (2015) Audiovisual quality assessment and prediction for videotelephony. Springer, Berlin. doi:10.1007/978-3-31914166-4

3. Duncanson JP (1969) The average telephone call is better than the average telephone call. Public Opin Q 33(1):112-116

4. Egger S, Hossfeld T, Schatz R, Fiedler M (2012) Waiting times in quality of experience for web based services. In: 4th International workshop on quality of multimedia experience (QoMEX), IEEE, Yarra Valley, VIC, pp 86-96. doi:10.1109/QoMEX.2012.6263888

5. ETSI (2011) Speech and multimedia transmission quality (STQ); Estimating Speech Quality per Call (V1.4.1)

6. Geerts D, De Moor K, Ketyko I, Jacobs A, Van den Bergh J, Joseph W, Martens L, De Marez L (2010) Linking an integrated framework with appropriate methods for measuring QoE. In: 2nd International workshop on quality of multimedia experience (QoMEX), IEEE, Trondheim, pp 158-163. doi:10.1109/ QOMEX.2010.5516292

7. Gros L, Chateau N (2001) Instantaneous and overall judgements for time-varying speech quality: assessments and relationships. Acta Acust Unit Acust 87(3):367-377

8. Gros L, Chateau N, Busson S (2004) Effects of context on the subjective assessment of time-varying speech quality: listening/conversation, laboratory/real environment. Acta Acust Unit Acust 90(6): 1037-1051

9. Guse D (2016) Multi-episodic perceived quality of telecommunication services. $\mathrm{PhD}$ thesis, Technische Universität Berlin. doi:10.14279/depositonce-5499

10. Guse D, Möller S (2013) Macro-temporal development of QoE: impact of varying performance on QoE over multiple interactions. In: Proceedings of AIA-DAGA conference on acoustics, Deutsche Gesellschaft für Akustik, Merano, vol 46, pp 452-455

11. Guse D, Weiss B, Möller S (2014) Modelling multi-episodic quality perception for different telecommunication services: first insights. In: 6th International workshop on quality of multimedia experience (QoMEX), IEEE, pp 105-110. doi:10.1109/QoMEX. 2014.6982304

12. Guse D, Wunderlich A, Weiss B, Möller S (2016) Duration neglect in multi-episodic perceived quality. In: 8th International conference on quality of multimedia experience (QoMEX)

13. Hamberg R, de Ridder H (1999) Time-varying image quality: modeling the relation between instantaneous and overall quality. SMPTE J 108(11):802-811. doi:10.5594/J04337

14. Hands DS, Avons SE (2001) Recency and duration neglect in subjective assessment of television picture quality. Appl Cognit Psychol 15(6):639-657. doi:10.1002/acp.731

15. Hogarth RM, Einhorn HJ (1992) Order effects in belief updating: the belief-adjustment model. Cogn Psychol 24(1):1-55. doi:10. 1016/0010-0285(92)90002-J

16. Hoßfeld T, Biedermann S, Schatz R, Platzer A, Egger S, Fiedler $M(2011)$ The memory effect and its implications on web QoE modeling. In: Proceedings of the 23rd international teletraffic congress, pp 103-110

17. ITU-T Recommendation P800 (1996) Methods for subjective determination of transmission quality (08/1996). International Telecommunication Union

18. ITU-T Recommendation P805 (2007) Subjective evaluation of conversational quality (04/2007). International Telecommunication Union
19. ITU-T Recommendation P851 (2003) Subjective quality evaluation of telephone services based on spoken dialogue systems (11/2003). International Telecommunication Union

20. ITU-T Recommendation P863 (2014) Perceptual objective listening quality assessment (09/2014). International Telecommunication Union

21. Jekosch U (2005) Voice and speech quality perception. Signals and communication technology. Springer, Berlin. doi:10.1007/3540-28860-0

22. Kahneman D (2000) Experienced utility and objective happiness: a moment-based approach. In: Kahneman D, Tversky A (eds) Choices, value and frames, vol 1. Cambridge University Press, Russel Sage Foundation, pp 673-708

23. Kahneman D, Fredrickson BL, Schreiber CA, Redelmeier DA (1993) When more pain is preferred to less: adding a better end. Psychol Sci 4(6):401-405

24. Karapanos E, Zimmerman J, Forlizzi J, Martens JB (2009) User experience over time: an initial framework. In: Proceedings of the SIGCHI conference on human factors in computing systems, ACM, New York, NY, USA, CHI '09, pp 729-738. doi:10.1145/ 1518701.1518814

25. Kujala S, Roto V, Väänänen-Vainio-Mattila K, Karapanos E, Sinnelä A (2011) UX curve: a method for evaluating long-term user experience. Interact Comput 23(5):473-483. doi:10.1016/j. intcom.2011.06.005

26. Köster F, Guse D, Wältermann M, Möller S (2015) Comparison between the discrete ACR scale and an extended continuous scale for the quality assessment of transmitted speech. In: Proceedings of AIA-DAGA conference on acoustics, Deutsche Gesellschaft für Akustik, Nuremburg, vol 48, pp 150-153

27. Lewcio B (2014) Management of speech and video telephony quality in heterogeneous wireless networks. Springer, Berlin. doi:10.1007/978-3-319-02102-7

28. McNeilly KM, Russ FA (1989) Errors in recall and judgment: the effect of information availability. J Gen Psychol 116(3):285-295. doi:10.1080/00221309.1989.9917762

29. Möller S, Bang C, Tamme T, Vaalgamaa M, Weiss B (2011) From single-call to multi-call quality: a study on long-term quality integration in audio-visual speech communication. In: 12th Annual conference of the international speech communication association, ISCA, Florence, pp 1485-1488

30. Ninassi A, Meur OL, Callet PL, Barba D (2009) Considering temporal variations of spatial visual distortions in video quality assessment. Select Topic Signal Process IEEE J 3(2):253-265. doi:10.1109/jstsp.2009.2014806

31. Raake A (2006) Speech quality of VoIP: assessment and prediction. Wiley, Chichester. ISBN 978-0-470-03060-8

32. Raake A, Egger S (2014) Quality and quality of experience. In: Möller S, Raake A (eds) Quality of experience. Springer, Berlin, pp 11-33. doi:10.1007/978-3-319-02681-7

33. Reiter U, Brunnström K, De Moor K, Mohamed-Chaker L, Pereira M, Pinheiro A, You J, Zgank A (2014) Factors influencing quality of experience. In: Möller S, Raake A (eds) Quality of experience. Springer, Berlin, pp 55-72. doi:10.1007/978-3-31902681-7

34. Rosenbluth JH (1998) Testing the quality of connections having time variant impairments. ITU-T Delayed Contribution D064

35. Roto V, Law E, Vermeeren A, Hoonhout J (eds) (2011) User experience white paper: bringing clarity to the concept of user experience

36. Sackl A, Casas P, Schatz R, Janowski L, Irmer R (2015) Quantifying the impact of network bandwidth fluctuations and outages on Web QoE. In: 7th international workshop on quality of multimedia experience (QoMEX), IEEE, Pylos-Nestoras, pp 1-6. doi:10.1109/QoMEX.2015.7148078 
37. Schacter DL, Chiao JY, Mitchell JP (2003) The seven sins of memory: implications for self. Ann N Y Acad Sci 1001:226-239. doi:10.1196/annals.1279.012

38. Schatz R, Egger S, Masuch K (2012) The impact of test duration on user fatigue and reliability of subjective quality ratings. J Audio Eng Soc 60(1):63-73

39. Shaikh J, Fiedler M, Paul P, Egger S, Guyard F (2013) Back to normal? Impact of temporally increasing network disturbances on QoE. In: Globecom Workshops (GC Workshops), IEEE, pp 1186-1191. doi:10.1109/GLOCOMW.2013.6825154

40. Strohmeier D, Mikkola M, Raake A (2013) The importance of task completion times for modeling web-QoE of consecutive web page requests. In: 5th International workshop on quality of multimedia experience (QoMEX), IEEE, Klagenfurt am Wörtersee, pp 38-39. doi:10.1109/QoMEX.2013.6603203
41. Tulving E (1972) Episodic and semantic memory. In: Tulving E, Donaldson W (eds) Organization of memory. Academic Press, New York, pp 381-402. ISBN 978-0127036502

42. Wechsung I, De Moor K (2014) Quality of experience versus user experience. In: Möller S, Raake A (eds) Quality of experience. Springer, Berlin, pp 35-54. doi:10.1007/978-3-319-02681-7

43. Weiss B, Möller S, Raake A, Berger J, Ullmann R (2009) Modeling call quality for time-varying transmission characteristics using simulated conversational structures. Acta Acust Unit Acust 95(6):1140-1151. doi:10.3813/AAA.918245

44. Weiss B, Guse D, Möller S, Raake A, Borowiak A, Reiter U (2014) Temporal development of quality of experience. In: Möller S, Raake A (eds) Quality of experience. Springer, Berlin, pp 133-147. doi:10.1007/978-3-319-02681-7 